\title{
Reference system for somatic cell counting in milk
}

\author{
Silvia Orlandini $\cdot$ Harrie van den Bijgaart
}

Received: 18 January 2011/ Accepted: 16 June 2011 / Published online: 29 June 2011

(c) The Author(s) 2011. This article is published with open access at Springerlink.com

\begin{abstract}
With some analytical parameters, certified reference materials are lacking and the reference method shows limited performance. Somatic cell counting in milk is a clear example. It is one of the most frequently performed measurements, estimated at over 500000000 tests/year world wide. It serves as an indicator for the udder health status of lactating animals, is relevant in food legislation, in payment of raw milk, and also has a considerable impact for farm management and animal-breeding programs. The analytical performance of nowadays fluoro-opto-electronic routine methods in terms of precision is superior to the reference method based on microscopy. Laboratories have therefore adopted various solutions for anchoring their counting level. It is there that a reference system approach can serve to optimally safeguard comparability of routine testing results in laboratories world wide. A reference system is characterized as a systematically developed anchoring system that is fed by different types of information from various sources, that is, measurements on reference materials, reference method analysis, and routine method results. A joint Project Group of the International Dairy Federation and the International Committee on Animal Recording has been given
\end{abstract}

Presented at the conference "Future of Reference Materials", November 2010, Geel, Belgium.

This study is conducted on behalf of the IDF/ICAR Project Group on Reference System for Somatic Cell Counting in Milk.

S. Orlandini $(\bowtie)$

Laboratorio Standard Latte, A.I.A., V. dell'Industria 24, 00057 Maccarese-Roma, Italy

e-mail: orlandini.s@aia.it

H. van den Bijgaart

Qlip N.V., Oostzeestraat 2a, 7202 CM Zutphen, The Netherlands

e-mail: bijgaart@qlip.nl the task to design a workable global reference system for somatic cell counting in milk. This paper describes the structure and the functioning of such a reference system, a plan for the implementation and the responsibilities of the involved stakeholders in safeguarding its functioning. After approval in IDF and ICAR, the resulting proposal is to be offered for adoption by concerned governmental and nongovernmental bodies worldwide.

Keywords Reference system - Raw milk analysis . Somatic cell count - SCC . Reference method . Routine method $\cdot$ Certified reference material

\section{Introduction}

The milk somatic cell count (SCC) is an important indicator of the udder health status of lactating mammals. Somatic cells excreted with milk include different types of white blood cells and some epithelial cells. The cellular composition and the concentration of cells change dramatically during periods of inflammation. An increase in SCC is regarded as the primary indicator of inflammation of the mammary gland [1].

SCC is therefore relevant in food legislation [2] and in payment of ex-farm raw milk, where it serves as a quality parameter. When measured with individual animals, it also has a major impact on farm management and breeding programs.

As a result, the determination of the somatic cell count is one of the most frequently performed measurements in dairy laboratories worldwide, estimated at more than 500000000 analyses per year.

SCC data are nowadays obtained almost exclusively through the application of automated fluoro-opto-electronic 
counting. Guidance on this application is available through ISO 13366-2IIDF 148-2 [3]. Part of the guidance is on calibration and calibration control. However, certified reference materials for SCC are lacking. Laboratories calibrate with reference materials that are different in matrix and range of concentration. A recent questionnaire [4] showed that these materials consist of either raw milk or heat-treated milk with natural somatic cells but in some cases also with added cells from other sources. The ranges provided are different, for example, from 200000 to 500000 cells $/ \mathrm{mL}$ or from 50000 to 1000000 cells $/ \mathrm{mL}$ for cows milk. Declared shelf life is from 7 to 180 days, depending on heat or preservation treatment. The characterization process of these reference materials is not homogeneous. The assigned values may derive wholly or partly from the application of the reference method, which is a direct microscopic somatic cell count according to ISO 13366-1IIDF 148-1 [5] and/or from routinely operated fluoro-opto-electronic method measurements.

In practice, many routine testing laboratories rely on these reference materials and their assigned values for safeguarding their counting level. Others base the calibration of their instruments on the performance in proficiency testing, and some rely on the standard settings of the instrument manufacturer.

The reason for not fully relying on the reference method is that it has some serious pitfalls and inherent weaknesses. It is felt that a reference system approach can overcome these shortcomings to a large extent and serve to better safeguard comparability in routinely operated somatic cell counting worldwide. A reference system is characterized as a systematically developed anchoring system that is fed by different types of information from various sources, that is, from reference materials, reference method analysis, routine method results, and proficiency testing results of laboratories operating in a laboratory network structure [6].

In this paper, it is explained how a joint project group of the International Dairy Federation and the International Committee on Animal Recording is trying to design a workable reference system for somatic cell counting in milk with a plan for its implementation.

\section{Reference method "versus" automated fluoro-opto-electronic methods for SCC}

The basic method for direct microscopic somatic cell counting (DMSCC) was described in 1910 by Prescott and Breed [7] and later modified by Newman-Lampert and Lewowitz-Weber cited in [3] and [8]. DMSCC is based on the counting of cell nuclei after staining, thereby using an optical microscope or a fluorescence microscope. The number of cells per milliliter of milk is obtained through multiplication of the counted number of cells in a defined viewing area with a working factor. DMSCC still serves as reference method for calibration and control purposes and is referred to as such in official regulations [9].

The widely adopted fluoro-opto-electronic methods are based on the same principle, but standardization and automation has resulted in a more robust counting process. A predetermined amount of test sample is mixed with a buffer and a stain solution and is fed to an object plane. That may be either the top surface of a vertical rotating disc (disc cytometry) or a capillary flow cell (flow cytometry). Each stained particle observed with a built-in fluorescence microscope produces an electrical pulse that is filtered, amplified, and recorded. The resulting pulse height distribution is electronically processed, whereby discrimination is made between noise signals and pulses that are attributed to stained somatic cells. The discriminator level can either be fixed or dynamic. With some instruments, it is possible to automatically adapt the duration of counting to the concentration of cells, for example, longer counting with lower number of cells. This is favorable for the precision of the measurements in the lower counting range.

Table 1 summarizes some critical positive $(+)$ and negative $(-)$ characteristics of the reference method and the routinely operated fluoro-opto-electronic methods.

The limitations of the reference method can be illustrated with the results of a collaborative study conducted in 2005 [10]. It was demonstrated that even with trained and experienced analysts, provided with an optimized description and pictures on "what to count and not to count" [5], analyst and laboratory effects were still substantial. The training and the skill of the analyst is to be considered as the main critical success factor for a proper performance of the reference method. It is therefore advised to execute the reference method with more analysts, preferably in more than one laboratory in order to reach for an optimal accuracy of resulting reference values.

Table 1 Comparison of reference method and fluoro-opto-electronic methods for SCC in milk

\begin{tabular}{ll}
\hline Reference method & Fluoro-opto-electronic methods \\
\hline+ Standardized & + Standardized guidance \\
(ISO 13366-1IIDF 148-1) & (ISO 13366-2IIDF 148-2) \\
- Historical method & + High throughput \\
- Varying visual appearance & + Fast (up to 500 samples/h) \\
of the measurand & \\
- Human subjectivity & + User-friendly \\
- Time consuming & + Low cost with high number \\
& of test samples \\
- Tedious & + Good precision \\
- Unsatisfactory precision & - No certified reference material \\
& available \\
\hline
\end{tabular}


Table 2 Precision data on the reference method and fluoro-optoelectronic methods for SCC (all data in $1000 / \mathrm{mL}$ ) in milk as interpolated from ISO 13366IIDF 148 parts 1 and $2[3,5]$

\begin{tabular}{lccccr}
\hline & Mean & $s_{\mathrm{r}}$ & $s_{\mathrm{R}}$ & $r$ & \multicolumn{1}{c}{$R$} \\
\hline Reference method & 245 & 38 & 41 & 107 & 114 \\
& 679 & 69 & 78 & 192 & 218 \\
Fluoro-opto-electronic methods & 245 & 13 & 20 & 36 & 57 \\
& 679 & 21 & 40 & 55 & 112
\end{tabular}

$s_{\mathrm{r}}$ standard deviation of repeatability, $s_{R}$ standard deviation of reproducibility, $r$ repeatability, $R$ reproducibility

Frequent execution of the method and participation in interlaboratory trials are considered essential measures to safeguard a comparable counting level [2].

Precision figures as obtained when following detailed standardized method descriptions [3,5] are listed in Table 2. Values for repeatability and reproducibility are dependent on the counting level, but are at all levels much more favorable for fluoro-opto-electronic methods than for the reference method.

It is clear that maintaining a stable counting level at routinely operated instruments through frequent calibration against the reference method is problematic with SCC. Worldwide comparability presently relies on:

- the well functioning and the proper operation of fluoroopto-electronic counters;

- a timely provision of reference materials with assigned values through reference material providers. However, reference materials are different in nature and in how assigned values are established. Only some providers interrelate the counting results on their material with those on material from other providers through intercomparison and/or participation in proficiency testing;

- participation of routine testing laboratories in proficiency testing to evaluate the precision and the accuracy with SCC.

\section{Joint IDF/ICAR initiative to develop a global reference system approach}

A joint project group of the International Dairy Federation (IDF, http://www.fil-idf.org) and the International Committee on Animal Recording (ICAR, http://www.icar.org) has been given the task to design a workable reference system for somatic cell counting in milk with a plan for its implementation. The final goal is to have a broad supported system implemented that optimally safeguards comparability in routine somatic cell counting world wide and is recognized as such by regulatory bodies, competent authorities, and other stakeholders. A more extensive explanation on the background and aims was provided by Baumgartner [6].

\section{Architecture of the reference system}

Discussions about how a reference system for somatic cell counting in milk could look like have been culminated in the scheme presented in Fig. 1.

Key aspects are:

- a suitable material for creating a certified reference material. The type and properties of the reference material should be optimized, preferably based on information from present providers and experiences in the application of these materials in terms of representativeness (commutability), stability, and shelf life.

- standardized methods. This is achieved for already through the two parts of ISO 13666'IDF 148 [3, 5].

- a procedure for collecting data from both reference method analysis and routine method analysis on the reference material. The limited precision of the reference method can be overcome by also taking into account results from correctly calibrated and wellcontrolled automated instruments. This feedback mechanism will help to smoothen the variations in subsequent batches that otherwise would likely occur due to the limited precision of the reference method.

- a system for scoring the competence of laboratories. The foregoing unavoidably asks for an objective judgement or score of the competence of those laboratories that participate in the assignment of reference values, both laboratories applying the reference method and those applying an automated routine method. This can be based on the performance of the concerned laboratories in proficiency trials.

- a system for scoring the quality of proficiency trials. Not all proficiency trials are the same. Proficiency trials may differ in the number of samples, in the range of the values, in the matrix, in the methods applied and in the number of participants. All these aspects have to be translated into an objectivated judgement or score.

- a data processing model for calculating and assigning reference values to the reference material. This model will be based on a probabilistic approach, which is presently subject to development and verification. The approach will allow to score the different proficiency tests and the performance therein of participating laboratories. Based on that, the relative weight of counting results from contributing laboratories in the overall assignment of reference values to the reference material can be determined. 
Fig. 1 Scheme of a reference system for somatic cell counting in milk
Criteria for

competence

of contributing labs
COORDINATOR

Criteria for

competence of contributing labs
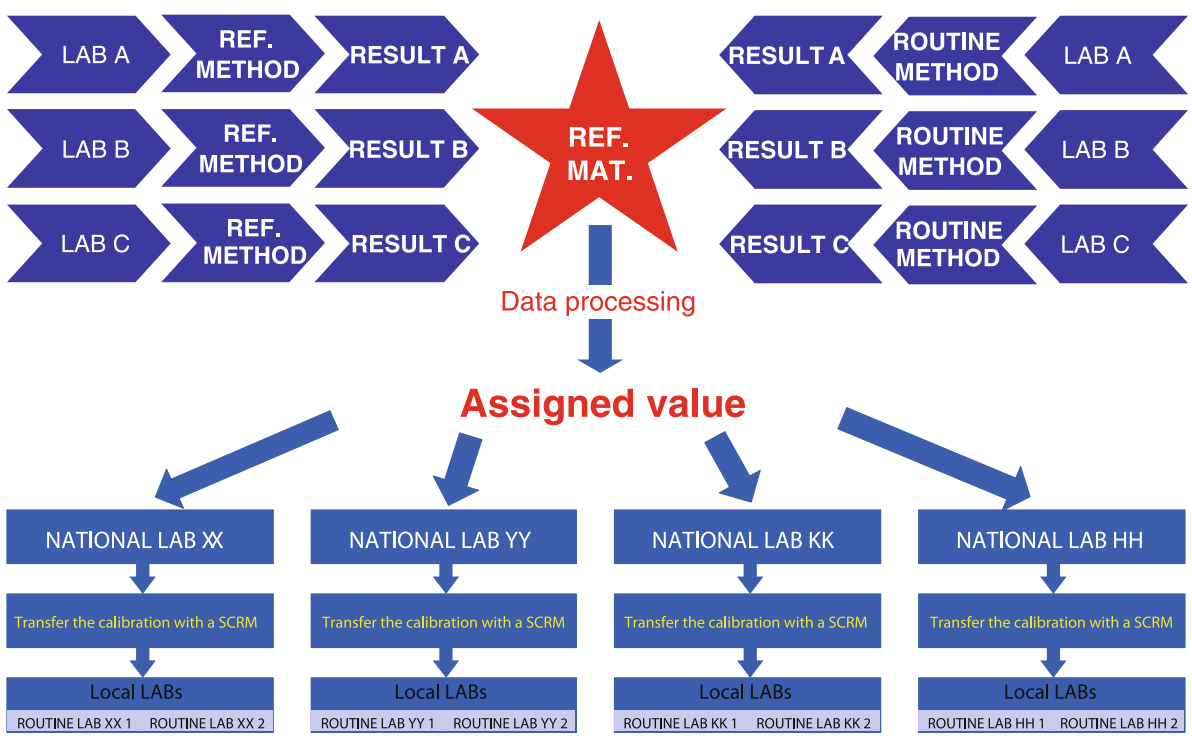

- secondary reference material (optional). Where the amount of the aforementioned certified reference material may be limited, there will be secondary reference materials needed to transfer the international 'level of equivalence' to local routine laboratories. An internationally standardized procedure for the preparation of these materials and the assignment of reference values should be strived for.

- a coordinator. Having a coordinator is a prerequisite for the functioning of this reference system for somatic cell counting. The concerned entity should act as an independent supervisor and promote a proper application of the principles and procedures.

This scheme is to be regarded as a target. Many small or larger-scale anchoring systems are already existent worldwide, as became apparent from recent questionnaires. It is felt that a bottom-up approach provides the most practicable trajectory in reaching for the final goal. In such an approach, it is key to realize further interlinkage between existing anchoring systems, see Fig. 2. In the first phase, the interlinkage can be created by having the same materials counted in different circles and achieving traceable

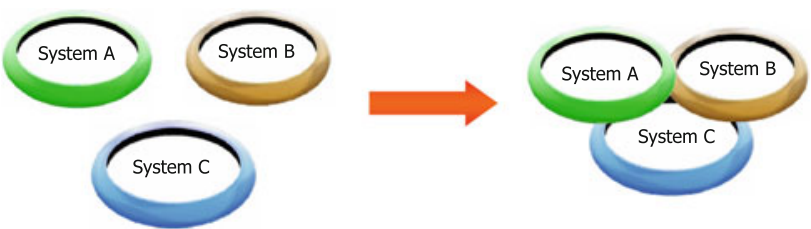

Fig. 2 Applying a bottom-up approach through interlinkage of existing anchoring systems alignment at a limited scale. Sharing and spreading of information on optimized procedures for the preparation of reference materials will promote harmonization on this aspect. These interlinked existing systems can also serve for the intended pilot study, in which the functioning of the aforementioned scoring systems and data-processing models can be tested and demonstrated before the plan is offered for adoption.

\section{Achievements thusfar}

The strong motivation for the project is demonstrated by the extensive participation in the IDF/ICAR project group with 23 representatives from 15 countries and three continents. The group has reached agreement on the target concept as presented in Fig. 1 and a stepwise bottom-up approach through interlinkage of existing anchoring systems. Table 3 lists the progress with each of the key aspects mentioned.

At the start of the project, communication on the aims of the project to the stakeholders was identified as an overarching critical success factor for their motivation. Therefore, a communication plan was built. The communication is and will be oriented toward both the analytical stakeholders (laboratories, reference material providers) as well as other bodies (regulatory, metrological, animal health and authorities bodies,). The aims, the plan, and the progress for the project are communicated through the ICAR and IDF Web sites, newsletters, papers, and presentations. 
Table 3 Progress on key aspects for the development of a reference system

\begin{tabular}{|c|c|}
\hline Key aspect & Progress as per end 2010 \\
\hline Suitable certified reference material & $\begin{array}{l}\text { Present suppliers of reference material have willingly provided } \\
\text { information on their material and practices through a questionnaire. } \\
\text { Further information on the use of reference material and calibration } \\
\text { practices is presently sought from routine laboratories } \\
\text { Requirements for reference materials are summarized and exchange } \\
\text { on best practices is ongoing }\end{array}$ \\
\hline Standardized methods & These are available $[3,5]$ \\
\hline Procedure for collecting analytical data on reference material & To be developed \\
\hline System for scoring competence of laboratories & $\begin{array}{l}\text { Will be based on a probabilistic approach, under development } \\
\text { by statistical experts }\end{array}$ \\
\hline System for scoring quality of proficiency trials & $\begin{array}{l}\text { Will be based on a probabilistic approach, under development } \\
\text { by statistical experts }\end{array}$ \\
\hline $\begin{array}{l}\text { Data-processing model for calculating and assigning } \\
\text { reference values }\end{array}$ & To be developed \\
\hline Secondary reference material & To be derived from guidance on certified reference material \\
\hline Coordinator & To be proposed with the final plan \\
\hline
\end{tabular}

\section{Next steps}

Next steps in the project are listed in Table 3. The focus for 2011 will be on the development of the scoring systems and the execution of a pilot study in which the developed approach will be tested.

\section{Concluding remarks}

Somatic cell counting in milk is a clear example of a situation where comparability of measurement results is suboptimal. The reference method has some distinct drawbacks and certified reference materials are lacking.

A joint initiative of IDF and ICAR is aimed at preparing a plan for a reference system approach. Such an approach is thought to optimally safeguard comparability in milk somatic cell counting and will at the same time allow individual laboratories to demonstrate traceability of their results.

The concerned IDF/ICAR Project Group has thus far experienced broad support from stakeholders worldwide. The critical points for a reference system approach have been identified and are presently being addressed:

- Willingness of laboratories to cooperate in a laboratory network structure and to share information and experiences. This cooperation is expected to promote alignment between laboratories through a learning effect.

- An objective system for the scoring of the quality of operational proficiency trials.

- An objective system for the scoring of the competence of laboratories.
- Consensus on the optimal preparation of reference material, the procedure for assigning reference values and the control on the subsequent transfer of reference material and reference data to routine laboratories.

- Support for the reference system approach from both the concerned laboratories as well as from regulatory bodies and other involved stakeholders.

After further detailing, the approach will be tested in a pilot study during the course of 2011-2012. This explorative study on the feasibility will focus on some already existing and interlinked anchoring systems for somatic cell counting in milk. These can serve as crystallization points for a gradual expansion to a global reference system. The outcome of the pilot study will be combined with other relevant information in a proposal for a robust reference system for somatic cell counting in milk. This will be offered for adoption by the stakeholders and concerned governmental and non-governmental bodies during the course of 2012-2013.

Acknowledgments The continued and valuable input of the members of the IDF/ICAR Project Group is gratefully acknowledged.

Open Access This article is distributed under the terms of the Creative Commons Attribution Noncommercial License which permits any noncommercial use, distribution, and reproduction in any medium, provided the original author(s) and source are credited.

\section{References}

1. Hogeveen H (ed) (2005) Mastitis in dairy production, current knowledge and future solutions. Wageningen Ac. Publication, Wageningen 
2. Regulation (EC) No 853/2004 of the European Parliament and of the Council of 29 April 2004 laying down specific hygiene rules for on the hygiene of foodstuffs. Official Journal of the European Union. 2004. 139/55. Annex II, section IX

3. ISO 13366-2IIDF 148-2:2006 Milk-enumeration of somatic cells, Part 2-guidance on the operation of fluoro-opto-electronic counters

4. Newsletter 2 of the IDF/ICAR Project Group on Reference system for somatic cell counting in milk. http://www.idfdairy laboratories.org/Public/dairylabs/BodyPage.php?ID=3800. Nov 2010

5. ISO 13366-1IIDF 148-1:2008 Milk-enumeration of somatic cells, Part 1-microscope method (reference method)

6. Baumgartner C (2008) Architecture of reference systems, status quo of somatic cell counting and concept for the implementation of a reference system for somatic cell counting. Bull IDF 427:15
7. Prescott SC, Breed RS (1910) The determination of the number of body cells in milk by a direct method. J Infect Dis 7:632

8. Shalm OW, Lasmanis J (1968) The leukocytes: origin and functioning with mastitis. J Am Vet Med Ass 153:1688

9. Commission Regulation (EC) No 1664/2006 of 6 November 2006 amending Regulation (EC) No 2074/2005 as regards implementing measures for certain products of animal origin intend for human consumption and repealing certain implementing measures. Official Journal of the European Union. Annex via chapter $1,26 / 33$

10. Orlandini S, Lattanzi L, Toscano AM, Paggi U (2008) Interlaboratory collaborative study on the reference method for somatic cell counting in cows milk ISO 13366-1'IDF 148-1. Bull IDF $427: 2$ 